\title{
IMPACT OF RESPIRATORY TRACT INFECTIONS ON DEVELOPMENTAL SKILLS IN CHILDREN WITH DOWN SYNDROME
}

\author{
R.H.J. Verstegen ${ }^{1}$, H.B.M. van Gameren-Oosterom ${ }^{2}$, M. Fekkes ${ }^{2}$, E. Dusseldorp ${ }^{2}$, E. de Vries ${ }^{1}$, J.P. van \\ Wouwe ${ }^{2}$ \\ ${ }^{1}$ Pediatrics, Jeroen Bosch Hospital, 's-Hertogenbosch, ${ }^{2}$ Child Health, Netherlands Organization for Applied \\ Scientific Research TNO, Leiden, The Netherlands
}

Background and aims: Recurrent respiratory tract infections (RRTI) have a significant impact on the developmental age of children with Down Syndrome (DS). Since RRTI are related to impaired hearing we proposed that verbal skills are more influenced compared to perceptual-performance, quantitative, memory and motor development in these children.

Methods: During a 3-year period, all members of the Dutch DS Foundation with an 8-year-old child with DS were asked to participate in this study. If parents agreed, a psychological assistant administrated the McCarthy Scales of Children's Abilities. Based on parental report, the children were divided into increased RRTI (RRTI+) and no increased RRTI (RRTI-). Linear regression analyses were performed to assess the effect of RRTI on the outcomes and the interaction with impaired hearing, adjusted for confounders.

Results: Compared to RRTI- children ( $n=176$; missing data $n=12)$, RRTI+ children $(n=149,46 \%)$ showed lower mean developmental age (3.67 vs. 4.08 years). Impaired hearing was more common in RRTI+ (44\% vs $18 \%$, p-values $<0.001$ ). RRTI+ children had lower scores on all scales (verbal, perceptual-performance, quantitative, memory and motor development; p-values $<.001)$ with equal effect sizes $\left(\mathrm{f}^{2}=.03-.04\right)$. Interaction with impaired hearing was not observed.

Conclusions: RRTI are related to delayed development in 8-year-old DS children. Although hearing loss is a common problem in RRTI+ children with DS, it does not lead to a more profound decrease in verbal skills compared to other domains of development. Since RRTI are potentially preventable, further studies should focus on causes and improved treatment of RRTI in children with DS. 